\title{
17. THE MOON (LA LUNE)
}

PRESIDENT: S. K. Runcorn.

VICE-PRESIDENTS: E. Anders, K. P. Florenskij.

ORGANIZING COMmITTEE: A. Dollfus, V. P. Dzhapiashvili, K. Koziel, J. D. Mulholland, J. A. O’Keefe, C. P. Sonett.

Since the General Assembly at Sydney very important advances have been made in our knowledge of the Moon, principally as the result of observations taken during the successful Apollo manned landings and the associated missions and the successful Luna flights in which Lunakhod carried out important traverses on the lunar surface and the automated study of the nature of samples by Luna 16 and 20. Laboratories of many nations took part in the analyses of samples through the distribution of samples by the United States through NASA, and the Soviet Union through the Academy of Sciences. The results of this work have been published in the Proceedings of the Lunar Science Conferences 1-7 which have appeared as supplements 1-7, each of 3 volumes, of Acta Geochim. Cosmochim., the proceedings of a Moscow conference on Cosmochemistry of the Moon in May 1974 and the Royal Society discussion meeting of June 1975 on 'The Moon: a New Appraisal from Space Missions and Laboratory Analyses' to be published in Phil. Trans. Roy. Soc. and in many papers and review articles. Members of the commission have contributed to this unique scientific programme.

Professor Dollfus has supplied the following report:

Many important studies upon the Solar System objects are based upon the imaging of their surfaces. The IAU Planetary Photographs Center (IAUPPC) was established at Meudon Observatory in 1961 on the recommendation of IAU: ' . . to facilitate research on collections of photographic plates previously scattered in several Observatories'.

Originally, the IAUPPC at Meudon filed the collections of plates on planets only, as collected through the world from telescopic observations (about 5000 original negative plates and 33000 positive copies were available in 1975).

Since 1973, thanks to the cooperation with the Space Agencies, the Center extended its field to include most of the imaging documents on planets collected by spacecraft during space missions (see report to Commission 16, same volume). Now the field of the Center also includes the lunar surface.

On the Moon, IAUPPC now houses the large collections of original negative plates of Loevy and Puiseux and the Pic-du-Midi plates, the principal atlas on the Moon and the lunar maps of the LOC-LAC-LTO series etc. ... plus Soviet maps and the geological maps. All the Ranger films and pictures, and some Surveyor documents are available. Thanks to NASA, all the Orbiter I to $V$ data are filed. Copies of all the Apollo 11 to 17 imaging documents are also stored; they include the pictures taken in orbit (except the 'panoramic' series) and those taken at the lunar surface during EVA. Supporting documents on the lunar samples are also available.

As for the work on planets (see report Commission 16), the IAU Planetary Photographs Center is open to all qualified scientists wishing to conduct researches upon the lunar surface which involve photographs, maps, imaging documents or their direct analysis.

Dr. Th. Weimar, Chairman of the Working Group 'Figure and Rotation of the Moon' reports as follows.

The Working Group is made up of 12 members who are members of IAU and 8 consulting members (not IAU members).

From January 1973 to January 1975, five bulletins 'Bibliography' were published giving references of about 160 articles ( 60 on the figure, cartography; 20 on the rotation, 80 on the observations, orbital action ...). This is the same number as for the previous period. 
The titles of the articles indicate that the work done from 1973-1975 is of the same kind as for the period 1970-1973.

It is worth noting that the most recent observational techniques (photographs from space vehicles, photoelectricity, laser) coexist with the classical (visual observations of occultations, heliometer, Earth-based photographs). But it seems probable that within a few years the geometry, the libration and even the orbit of the Moon will be determined with great accuracy by the new methods which will supplant all the older ones. The 'Bibliography' of the Working Group gives a good outline of the people and institutes involved in these new techniques and the promising results being obtained.

Working Group 2 on the Physics, Chemistry and Geology of the Moon under its Chairman, Dr J. A. O'Keefe, has reviewed the status of the impact theory of the formation of the lunar craters. A full report of these discussions will be published in the scientific press. It suffices here to say that the characteristics of the lunar craters have convinced most scientists that impacts have been the cause of most lunar craters. Since the space missions most lunar scientists see in the regolith of the Moon the record of impacts of all sizes - from microscopic to gigantic ones for which there are no terrestrial analogies. Challenge by those who believe a substantial fraction are volcanic raises points which have perhaps been glossed over by the majority group and has pointed to the need to decide what constitutes diagnostic evidence to decide in any particular case between a volcanic or impact origin.

Dr Florenskij reports on work in the Soviet Union as follows:

I. The Astronomy Council of the Academy of Sciences of the U.S.S.R.

From the investigations of the lunar surface microstructure using spectral measurements in IR- and UV-rays from 'Zond-3', one can observe the bimodal structure (G. A. Leikin et al., Physics of the Moon and Planets, M., 1972). A morphometrical analysis of the visible side of the Moon by making use of the Mills catalogue has led to the supposition of the rough isostasy of the surface (G. A. Leikin et al., Astron Tsirk, No. 755, 1973). The method used was a statistical analysis of the craters's morphological characteristics. A meaningful difference of the characteristics for parts of the mare surface is revealed and their physical interpretation is suggested (G. A. Leikin et al., Astron. Vestnik 8, No. 2, 1974).

\section{The State Astronomical Institute}

The preparation of the third part of The Reverse Side Atlas of the Moon is completed. The Moon globus is published in the scale 1:10000 000. A unified catalogue of the base points on the visible side of the Moon was constructed (Yu. N. Lipskij, V. A. Nikonov, T. P. Skobeleva, $A$ Unified System of Selenodetic Coordinates from Nine Catalogues on the Visible Hemisphere of the Moon, Nauka, M., 1973). A photometrical investigation of the far side of the Moon was conducted on the basis of the Zond data (Yu. N. Lipskij et al. Kosm. Issled. 12, No. 5, 1974). A chart of the Moon sphere albedo was constructed with an $80 \%$ coverage of the Moon surface (V. V. Shevchenko, Astron. J. 51, No. 5, 1974). Photometrical investigations by 'Lunokhod-2' were conducted (Yu. N. Lipskij, V. V. Shevchenko, Works of the Conference on the Moon and Planets Cosmochemistry, Nauka, M., 1975).

\section{Astronomical Observatory Angelgardta}

The calculation of the Moon barycentrical heights on the basis of heliometrical observations (Gartving, 1890-1922). and Nefed'ev, 1946-1958, 1971-1974) was completed. Highly precise heliometrical measurements of 36 position points were conducted (1969-1975). A relief analysis of the far side of the Moon was conducted by studying limb photographs of Zond-8. An extensive fall was revealed in the south-east limb region.

IV. The Astronomical Observatory of Kazanskij State University of Ulianova-Lenina

Investigations of the rotation, figure and gravitational field of the Moon were conducted. The analysis of the coefficients of the gravitational field of the Moon was conducted on the basis of the Lunar Orbiter data (Sh. T. Habibullin, U. A. Chikanov, Astron. J. 49, No. 1, 1972). The method of comparison of the supporting points catalogue on the Moon surface was based on 
the theory of deformation of a continuous medium (Sh. T. Habibullin et al., The Moon 3,1972). The method of treatment of the observations and of the determination of the absolute coordinates of the craters for the composition of the 200 base points catalogue was worked out (Sh. T. Habibullin et al., The Moon 11, 1974).

V. Main Astronomical Observatory of the Academy of Sciences of the Ukrainian S.S.R. (Kiev, Goloseevo)

A summary of the selenodetical coordinates system, containing over 4500 base points on the visible side was constructed. The project of the international standardization of the selenodetic coordinate system was worked out (I. V. Gavrilov, V. S. Kisluk). An analysis of the Zond-8 photographs was conducted, the limb profile on the far side of the Moon near the $150^{\circ}$ west longitude was constructed, and a depression of $2-3 \mathrm{~km}$ depth was revealed. Limb measurements, based on photographs taken from the surface of the Earth, were made, and the absolute heights of the 960 points of the border zone were defined (A. S. Duma, L. N. Kizun).

VI. Astrophysical Institute of the Academy of Sciences of Kazakh S.S.R.

Calorimetrical investigation of selected regions of the Moon was conducted (N. V. Priboeva, Astron. Vestnik 7, No. 4, 1973).

VII. The Institute of the Earth Physics of the Academy of Sciences of the U.S.S.R.

Questions on the nature of mascons were considered (V. N. Iarkov, "A Report on the Conference on the Moon and Planets Cosmochemistry', M., 1974). The results of a series of works on the Moon's nature and origin were generalized (E. L. Ruskol, The Origin of the Moon, Nauka, M., 1975).

VIII. The Institute of Geology of the Academy of Sciences of the U.S.S.R.

A series of works on the Moon's geology was carried out under the leadership of Markov (The Volcanism and the Tectonics of the Moon, Nauka, M. 1974).

\section{The Central Institute of Geodesy, Aerial and Cartography}

The main rules on Moon mapping were wcoked out jointly with NASA (U.S.A.). The large scale topographic maps were built on the basis of Lunokhod-1 and Lunokhod-2 materials. A statistical analysis of the relief along the paths of the Lunokhods was conducted.

X. Moscow Institute of the Engineers of Geodesy, Aerial and Cartography

A map of the far side of Moon in a scale 1:1000000 on ten sheets covering the region of the Zond-8 survey was composed. A model of the anomalous gravitational field of the Moon containing 83 point masses was obtained on the basis of Lunar Orbiter data. The accuracy of this model was investigated.

XI. Abastumani Astrophysical Observatory of the Academy of Sciences of the Georgian SSR

A series of works on polarimetrical investigations of the Moon was carried out (V. P. Japiashvili et al., in the collection Physics of the Moon and Planets, Nauka, M., 1972). A sharp time change of the polarization in the Posidoni crater was revealed (V. P. Japiashvili, Astron. Vestnik 6, No. 1, 1972). An experimental sample of the Moon polarization map was published on the basis of the Polarovizornukh observations (V. P. Japiashvili, Astron. Tsirk. No. 788,1973$)$.

XII. The Institute of Cosmic Investigations of the Academy of Sciences of the U.S.S.R.

Work on preparation of the fundamental geodetic system was carried out (A. A. Gursh tein et al. Astron. J. 51, No. 4, 1974). The 'Map of the Moon' on both hemispheres in a scale 1:10000000 and 1:25000000 and the 'Topographic Map of the Moon in the Region of Action of Lunokhod 2' in a scale 1:50 000 were prepared and published.

A series of works on the problem of structure and evolution of the Moon's surface (K. P. Florenskij et al. in a collection A Modern Sight about the Moon, Nauka, M., 1972) and 
on the influence of the exogenic factors in the forming of the Moon surface (K. P. Florenskij, A. V. Ivanov, and A. T. Bazilevskij, Transactions of the Conference on the Cosmochemistry of the Moon and Planets, Nauka, M., 1975) was carried out. A treatment and investigation of the Lunokhod-2 pictures was conducted and morphological peculiarities were interpreted on the basis of this investigation (K. P. Florenskij et al., The Report of the Academy of Sciences of the U.S.S.R. 214, No. 1, 1974).

XIII. The Institute of Geochemistry and Analytical Chemistry of the Academy of Sciences of the U.S.S.R.

A complex investigation of the lunar soil samples delivered by 'The Moon-16' was conducted (A. P. Vinogradov et al., Lunar Soil from the Mare Fecunditatis, M., 1973). The composition of the gases emitted during solar heating of the Lunar regolith was shown from the soil sample, delivered by Luna 16. (Yu. A. Surkov et al. Transactions of the Conference on the Cosmochemistry of the Moon and Planets, Nauka, M., 1975). The lunar soil sample delivered from the continental region by Luna 20, was investigated (A. P. Vinogradov et al., Transactions of the Conference on the Cosmochemistry of the Moon and Planets, Nauka, M., 1975).

XIV. Kharkov State University

The work on the map of the optical characteristics of the visible Moon hemisphere surface was conducted in a scale 1:5000000 (N. N. Evsukov 'The Map of the Albedo of the Visible Lunar Hemisphere', 'Naukova dumka', Kiev, 1973, 'The Map of the Colour of the Visible Lunar Hemisphere', 'Naukova dumka', Kiev, 1973, 'The Photometrical Map of the Visible Lunar Hemisphere', 'Naukova dumka', Kiev, 1973) and a possible connection of the optical parameters with a chemical composition of the rocks was investigated.

S. K. RUNCORN

President of the Commission 